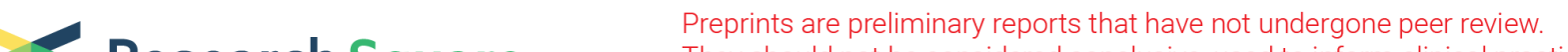 $\begin{array}{ll}\text { Research Square } & \text { They should not be considered conclusive, used to inform clinical practice, } \\ \text { or referenced by the media as validated information. }\end{array}$
}

\section{The Effect of Mildly Stimulated Cycle Versus Artificial Cycle On Pregnancy Outcomes In Overweight/Obese Women With PCOS Prior To Frozen Embryo Transfer: A Retrospective Cohort Study}

\section{Lu Guan}

Shandong University of Traditional Chinese Medicine

Shan Xiang

Shandong University of Traditional Chinese Medicine

\section{Haicui Wu}

Affiliated Hospital of Shandong University of Traditional Chinese Medicine

\section{Chaofeng Wei}

Shandong University of Traditional Chinese Medicine

\section{Conghui Pang}

Affiliated Hospital of Shandong University of Traditional Chinese Medicine

\section{Danqi Liu}

Shandong University of Traditional Chinese Medicine

\section{Xiaona Yu}

Shandong University of Traditional Chinese Medicine

Fang Lian ( $\sim$ lianfangbangong@163.com )

Shandong University of Traditional Chinese Medicine

\section{Research Article}

Keywords: Polycystic ovary syndrome, Frozen embryo transfer, Endometrial preparation, Artificial cycle, Mildly stimulated cycle, Overweight, Obesity,

Posted Date: January 20th, 2022

DOI: https://doi.org/10.21203/rs.3.rs-1161401/v1

License: (a) (1) This work is licensed under a Creative Commons Attribution 4.0 International License.

Read Full License 


\section{Abstract}

Background: Previous studies have shown that frozen embryo transfer (FET) resulted in increased live birth rates (LBR) and reduced the risk of ovarian hyperstimulation syndrome (OHSS) than did fresh embryo transfer in women with polycystic ovary syndrome (PCOS). In addition, overweight/obese women with PCOS are at increased risk of subfertility and complications of pregnancy, compared with normalweight women. The ovarian stimulation and artificial hormone regimes are the two more commonly used endometrial preparation protocols in PCOS patients. This retrospective study aims to compare the pregnancy outcomes of mildly stimulated cycles (mSTC) and artificial cycles (AC) prior to FET in overweight/obese women with PCOS.

Methods: A retrospective analysis was conducted in overweight/obese women with PCOS who underwent their first FET cycles from January 2018 to December 2020. Two endometrial preparation protocols were used: the mildly stimulated cycles $(\mathrm{N}=173)$ and the artificial cycles $(\mathrm{N}=507)$. All pregnancy outcomes were analyzed by Student's $t$-test, Chi-square $\left(\chi^{2}\right)$ statistics and multivariable logistic regression analyses.

Results: This study enrolled 680 cases of FET cycles. The mSTC group exhibited significantly higher LBR compared with the AC group (49.7\% vs. $41.0 \% ; P=0.046)$, while the rate of miscarriage was significantly lower $(6.4 \%$ vs. $23.0 \% ; P<0.001)$. No statistically significant differences were observed in positive pregnancy rate $(57.8 \%$ vs. $60.0 \%, P=0.618)$, clinical pregnancy rate $(54.3 \%$ vs. $55.6 \%, P=0.769)$, and ectopic pregnancy rate $(2.1 \%$ vs. $3.2 \%, P=0.860)$ between two groups. After adjusting for possible confounding factors, multivariate logistic regression analysis also yielded similar results.

Conclusions: For overweight/obese women with PCOS, mSTC-FET demonstrated a higher LBR and a lower pregnancy loss rate than that in the AC-FET. When considering the most cost-effective treatment with the least adverse effects on patients, the MSTC for FET endometrial preparation may be considered. To corroborate our findings, additional prospective randomized clinical trials with larger sample sizes are required.

\section{Background}

Polycystic ovary syndrome (PCOS) is the most common endocrine and metabolic disease in women of reproductive age, with an incidence as high as 10\% [1]. PCOS is often concomitant with obesity, oligo-or amenorrhea, hormonal abnormality, and infertility. Obesity is closely associated with PCOS and is critical in PCOS development and progression. A study from Spain revealed that PCOS might be more prevalent among overweight and obese women [2], but its incidence rate varied substantially across regions and ethnic groups [3]. In addition, several studies have indicated that PCOS disease status significantly increases the risk of obesity [4-6]. Menstrual disturbances were present in half of obese women with PCOS [4]. Both overweight and obesity significantly reduced the pregnancy rate [4] as well as increased the risk of miscarriage and preterm birth in PCOS patients [7]. In addition, they were associated with a 
high rate of pregnancy complications such as gestational diabetes mellitus and gestational hypertension [8].

PCOS is the most common cause of anovulatory infertility, accounting for approximately $80 \%$ [9]. When traditional treatment methods fail to produce satisfactory results, assisted reproductive techniques (ART) are considered final treatments for PCOS patients, including controlled ovarian hyperstimulation $(\mathrm{COH})$ and embryo transfer. With the refinement of vitrification techniques and ongoing adoption of single embryo transfer strategies, the number of frozen embryo transfer cycles has drastically increased [10]. Due to the increased use of ovulation induction drugs on PCOS, the risk of ovarian hyperstimulation syndrome (OHSS) in these patients increases. In addition to personalized ovulation induction protocols and single embryo transfer, a freeze-all policy is the better choice for PCOS patients seeking to reduce OHSS risk [11]. Previous studies have indicated that this freeze-all strategy significant increases pregnancy frequency rates and live birth rates (LBR) while also significantly reducing the risk of OHSS, which can occur in patients undergoing in vitro fertilization (IVF) and ovarian stimulation, potentially leading to death $[12,13]$. FET outcomes depend on many factors, including embryonic quality, endometrial receptivity, and their synchronization. Compared with fresh embryo transfer, FET is closer to the hormone environment of natural pregnancy [13], avoiding the adverse effects of controlled ovarian hyperstimulation on embryo-endometrium synchronization [14].

Different endometrial preparation regimes have been applied to increase endometrial receptivity in FET cycles, including natural, stimulated (STC), and artificial cycles (AC). In the natural cycle, endometrial preparation depends on endogenous steroid hormones produced by developing follicles, similar to natural physiological state. The optimal embryo transfer stage can be determined by monitoring the peak of endogenous luteinizing hormone or ovulation time. Endometrial preparation using a natural cycle may appear more acceptable and cost-effective due to the absence of injections. However, this method is only appropriate for patients with regular menstruation. It might be less convenient for PCOS patients due to the required early medication intervention to maintain regular menstrual periods and more monitoring to determine the appropriate day for embryo transfer. It might also be less convenient for centers due to reduced flexibility $[15,16]$. Therefore, the ovarian stimulation protocol and artificial hormonal endometrial preparation are the two most often used endometrial preparation regimens for PCOS patients. A metaanalysis including different regions and ethnic groups revealed that the prevalence of overweight and obesity in PCOS women ranged from $40.0 \%$ to $100 \%$ [5]. Furthermore, a retrospective study suggested that embryo implantation and live birth rates were significantly lower in overweight/obese women patients than in normal-weight women with PCOS, while the late abortion rate was significantly higher [17]. Previously, few retrospective analyses evaluated the pregnancy outcomes of overweight/obese women with PCOS after treatment using different endometrial preparation protocols. This study compares the efficacy of mildly stimulated cycle (mSTC) versus AC for endometrial preparation before FET in a retrospective study of PCOS overweight/obese patients.

\section{Materials And Methods}




\subsection{Study Design and Participants}

This retrospective study was performed at the Reproduction and Genetics Center of Integrated Traditional Chinese and Western Medicine at the Affiliated Hospital of Shandong University of Traditional Chinese Medicine. It was approved by the Reproductive Ethics Committees of the Center (ref approval no. SDTCM20201008). Because this was a retrospective investigation, patients were not asked to participate in the analysis. This study enrolled 680 cases of PCOS overweight/obese women undergoing IVF/ICSI (intracytoplasmic sperm injection) and their first FET cycles from January 2018 to December 2020. According to Rotterdam consensus [18], PCOS was diagnosed as fulfilling at least two of the three following items: 1) oligo-anovulation or anovulation; 2) clinical or biochemical signs of hyperandrogenism; and 3) polycystic ovarian morphology on ultrasound, as defined by at least one ovary with a volume $\geq 10 \mathrm{~cm}^{3}$ or $\geq 12$ follicles. Further inclusion criteria included participants aged between 20 and 38 years and body mass index (BMI) $\geq 25 \mathrm{~kg} / \mathrm{m}^{2}$. Exclusion criteria included the following: 1) other causes of hyperandrogenism and ovulation dysfunction; 2) A history of recurrent miscarriage; 3 ) severe endometriosis; 4) congenital uterine malformations; 5) karyotypic abnormalities; and 6) cycles canceled due to failure of embryo thawing and survival (Fig. 1).

\section{Figure 1.Workflow of study enrollment.}

\subsection{Ovarian stimulation and laboratory protocols}

All patients underwent one of the following two $\mathrm{COH}$ regimens: a flexible gonadotropin-releasing hormone antagonist ( $\mathrm{GnRH}$-ant) protocol or a standard $\mathrm{GnRH}$ agonist ( $\mathrm{GnRH}-\mathrm{a}$ ) long protocol. Briefly, patients in the flexible GnRH-ant protocol were injected daily with 150-225 IU recombinant FSH (Gonal-F, MerckSerono, Lyon, France) from day 2 or 3 of the menstrual cycle, with daily $0.25 \mathrm{mg} \mathrm{GnRH}$-ant (Cetrorelix, Merck Serono, Darmstadt, Germany) being initiated once the largest follicle was $>12-14 \mathrm{~mm}$ in size. For the standard $\mathrm{GnRH}-\mathrm{a}$ long protocol, patients underwent pituitary down-regulation using $\mathrm{GnRH}-\mathrm{a}$ (Triptorelin, Decapeptyl, Ipsen, France) during the luteal phase of the previous menstrual cycle, followed by an initial dose of 150 225 $\mathrm{U}$ recombinant FSH for ovulation induction. Additionally, gonadotropin doses were adjusted according to the ovarian response, as monitored via ultrasonography and measurement of serum sex steroids. When $\geq 2$ follicles measured $\geq 18 \mathrm{~mm}, 4000-10000$ IU human chorionic gonadotropin (hCG; Lizhu, Zhuhai, China) or $250 \mu \mathrm{g}$ of recombinant human choriogonadotropinalfa solution (Ovitrelle ${ }^{\circledR}, 250 \mu \mathrm{g}$, Merck) was administered to trigger final oocyte maturation. Oocyte retrieval was performed 34-36 $\mathrm{h}$ later. A conventional IVF/ICSI approach was employed to fertilize the harvested oocytes in light of the specifics of a given semen sample. According to clinical practice indication, IVF/ICSI procedures were either followed by fresh embryo transfer or a freeze-all strategy.

High-quality embryos that were not used for fresh embryo transfer were cryoprotected in a closed vitrification system. For cleavage-stage embryos (day 3 ), the quality was graded using Cummins criteria [19], and the best quality embryos (grade I-II) were selected for vitrification. Suboptimal day-3 embryos (grade III-IV) were placed in extended culture to the blastocyst stage. Day-5 embryo quality was evaluated 
based on the scoring system of Gardner and Schoolcraft [20], with embryos graded $\geq 3$ BB classified as high-quality blastocysts. All FET cycles include the transfer of up to two embryos. On the day of transfer,

all embryos were thawed, and any embryo with more than $50 \%$ blastomeres was considered transplanted. The vitrification and thawing procedures were performed as in previous reports [21].

\subsection{Endometrium preparation protocols}

\subsubsection{Mildly stimulated cycles}

In the mildly ovarian stimulation protocol, letrozole (LE; Jiangsu Hengrui Medicine Co., China) was administered orally for five consecutive days at a daily dose of $5 \mathrm{mg}$, initiating on day 3 of a spontaneous menstrual cycle or progesterone-induced withdrawal bleeding. After that, a daily dosage of human menopausal gonadotropin (hMG; Anhui Fengyuan Pharmaceutical Co., China) 37.5 75 IU was administered to stimulate follicle growth. Ultrasound monitoring and serum hormone analysis were performed from cycle day 10 onwards. Transvaginal ultrasound monitoring was performed every three days. Whenever the endometrial thickness was $\geq 7 \mathrm{~mm}$, and dominant follicle $\geq 17 \mathrm{~mm}$, with $E_{2}$ levels preferably $>150 \mathrm{pg} / \mathrm{mL}, \mathrm{HCG} 4000 \mathrm{IU}$ were injected for the final oocyte triggering. An injection of progesterone (Zhejiang Xianju Pharmaceutical Co., China), $40 \mathrm{mg}$ per day, was provided two days following HCG administration. Three or five days after progesterone administration, synchronized warm embryos are transferred. After 14 days of ovarian stimulation, if the endometrial thickness did not reach 7 $\mathrm{mm}$, the cycle was canceled regardless of the dominant follicle size.

\subsubsection{Artificial cycles}

In the artificial cycle, endometrial preparation includes sequential administration of estradiol valerate tablets (DELPHARM Lille S.A.S, France) and progesterone. On days 2-3 of the spontaneous or discontinued progesterone menstrual cycle, if endometrial thickness was less than $5 \mathrm{~mm}$ and serum estradiol level was less than $50 \mathrm{pg} / \mathrm{mL}$ assisted by vaginal ultrasound and blood monitoring, endometrial preparation was started by prescribing $4 \mathrm{mg}$ estradiol valerate daily for five days, followed by $6 \mathrm{mg}$. On the $13^{\text {th }}$ day of estradiol administration, an ultrasound scan was conducted to measure endometrial thickness. If endometrial thickness was $\geq 7 \mathrm{~mm}, 40 \mathrm{mg}$ daily progesterone injection was initiated. If endometrial thickness was less than $7 \mathrm{~mm}$, estradiol valerate tablets were increased to $8 \mathrm{mg}$ /day orally until the intima reached the appropriate thickness. Three or five days after progesterone administration, synchronized warm embryos were transferred. If the endometrial thickness remains inadequate, the cycle would be canceled. If a pregnancy is confirmed, luteal support cannot be withdrawn in mSTC and AC groups until ten weeks of gestation.

To date, few studies have demonstrated the ideal means of endometrial preparation in overweight/obese women with PCOS. The choice of endometrial preparation depends on patient and physician preference. However, frozen embryo transfer has been a standardized treatment for PCOS patients since 2012 at our reproduction center. 


\subsection{Outcome measures}

The primary outcome of the study was live birth rate (LBR), and the secondary outcomes included implantation rate, positive pregnancy rate, clinical pregnancy rate, endometrial thickness, ectopic pregnancy rate, and pregnancy loss rate. Implantation rates were determined via ultrasound-mediated assessment of how many gestational sacs were evident in a patient compared to the number of transferred embryos. Positive pregnancy was defined as a serum $\beta$-hCG level $\nabla 10 \mathrm{IU} / \mathrm{L}$ at 14 days after cleavage embryo transfer. If $\beta$-hCG assay yielded a positive result, clinical pregnancy was established as the presence of at least one gestational sac in the uterine cavity on ultrasound five weeks after FET. Live birth was defined as a live-born baby after $\geq 24$ gestational weeks. The pregnancy loss was defined as a loss of clinical pregnancy within 20 weeks. Ectopic pregnancy refers to abnormal pregnancy in which fertilized egg implants outside the uterine cavity and can be diagnosed by ultrasound, surgical visualization, or histopathology.

\subsection{Statistical analysis}

Statistical analysis was performed using Statistical Package for Social Sciences (SPSS) version 21.0 (SPSS Inc., Chicago, USA). The continuous variables were expressed as mean \pm standard deviations (SD) and were compared between groups using Student's $t$-test. The Chi-square $\left(\chi^{2}\right)$ statistics was conducted to compare categorical variables between two groups, and the results were expressed as numbers/percentages. Multivariate logistic regression analysis was utilized to examine the possible effects of the following known potential confounding factors on pregnancy outcomes of FET cycle, including age at FET, BMI, infertility duration, infertility type (primary or secondary), ovulation induction regimen (agonist regimen or antagonist regimen), gonadotropin (Gn) usage time, Gn dosage, oocytes retrieved, laboratory fertilization mode (IVF or ICSI), number of embryos transferred, embryo stage and quality at transfer, and endometrium thickness prior to FET. A $P$-value $<0.05$ was considered statistically significant.

\section{Results}

\subsection{Baseline and ART Characteristics of Patients}

The current study analyzed 680 FET cycles. Specifically, 173 (25\%) patients received mSTC, and 507 (75\%) patients underwent AC before FET. Patients' baseline characteristics are detailed in Table 1. No significant difference was observed between the two treatment groups regarding age, infertility duration, $\mathrm{BMI}$, or type of infertility. The baseline hormone profile at cryopreservation was similar between the two groups. The ovulation induction regimens used were agonist (496 cases, 72.9\%), antagonist (184 cases, $27.1 \%)$, laboratory IVF (573 cases, 85.7\%), and ICSI (107 cases, 14.3\%). In mean values, 22 oocytes were retrieved, and 7 embryos were frozen.

\subsection{Cycle Characteristics of FET}


As presented in Table 2, the mSTC group exhibits significantly greater endometrial thickness prior to FET $(10.10 \pm 1.94$ vs. $9.75 \pm 1.61, P=0.039)$. Most patients were transferred double embryos $(86.2 \%)$ of $\mathrm{D} 3$ stage (87.8\%).

\subsection{Pregnancy outcomes}

Our findings of pregnancy outcomes in these two different FET groups are described in Table 3. The LBR were $49.7 \%$ and $41.0 \%$ following mSTC-FET and AC-FET, respectively, reaching statistical significance $(P=0.046)$. Compared with AC group, those in the MSTC group exhibited a significantly lower miscarriage rate $(6.4 \%$ vs. $23.0 \% ; P<0.001)$. However, no statistical significance was observed between two groups in implantation rates, positive pregnancy, and clinical pregnancy. Moreover, the rates of ectopic pregnancy and twin pregnancies were similar between two groups.

After adjusting for the confounding factors between endometrial preparation regimes and pregnancy outcomes (Table 4), LBR remained consistently higher, and the miscarriage rate was still significantly lower following mSTC group. Furthermore, in the crude and adjusted models, the mSTC group was comparable to the AC group in terms of positive and clinical pregnancy rates.

\section{Table 1. Baseline characteristics of ovarian stimulation and IVF cycles.}

\section{Table 2. FET cycle characteristics between groups.}

\section{Table 3. Pregnancy outcomes between groups.}

Table 4. Binary logistics regression analysis with pregnancy outcomes as the influencing factor.

\section{Discussion}

Obesity and PCOS are closely related disorders with overlapping features, which may negatively impact pregnancy or neonatal outcomes [22]. So far, few studies have evaluated different preparation methods of endometrium before FET in overweight/obese women with PCOS. In this retrospective study of 680 overweight/obese women with PCOS undergoing FET, pregnancy outcomes of 173 stimulated cycles were retrospectively evaluated with reference to outcomes of 507 artificial cycles. We provided clinically relevant evidence suggesting that, compared with AC-FET group, the mSTC-FET group had significantly higher LBR and a significantly lower rate of pregnancy losses.

The abnormalities of basal hormone levels in PCOS patients have presented as high levels of androgens and $\mathrm{LH}$, as well as low levels of $\mathrm{FSH}$ and $\mathrm{E}_{2}$, which might have unpleasant effects on the development of follicles, ovulation, and endometrial receptivity, eventually leading to infertility. Potential mechanisms of obesity-related PCOS included insulin resistance and hyperinsulinemia, lipotoxicity secreted by excessive free fatty acids, adipokines produced by abnormal secretion of adipocytes, and inflammatory reaction induced by obesity [23]. Numerous factors influence the pregnancy outcomes of FET, such as the age of patients, the quality of embryos, endometrial receptivity, 
and so on, among which endometrial receptivity is one of the key factors. So far, there is no comparative study identifying the best protocol to prepare the endometrium in overweight/obese women with PCOS before FET. A large retrospective study of 1556 women with PCOS undergoing FET found that overweight and obese women achieved similar pregnancy outcomes, including implantation rates, clinical pregnancy, and live birth, as did normal-weight women when the quality of thawed embryos transferred was similar. It was concluded that obesity has a detrimental effect on embryo quality while not affecting endometrial receptivity or early implantation [22]. After adjusting for possible confounding factors, Zhang et al.'s large retrospective study confirmed our findings that letrozole-stimulated cycles (L-FET) had significantly higher LBR and lower pregnancy loss rate than AC-FET [24]. Another retrospective cohort study conducted in Japan by Tatsumi et al. indicated that letrozole stimulation during FET resulted in higher clinical pregnancy and LBR and a lower risk of miscarriage than substituted and natural cycles [25]. To corroborate the findings of Tatsumi et al., a more recent retrospective cohort study conducted in Israel concluded that ovulation induction using letrozole might induce normal ovulation and physiological FET, resulting in better pregnancy rates in FET treatments for PCOS patients [26]. A recent historical cohort analysis on women with PCOS found that ovarian stimulation (OS) protocol with low doses of HMG achieved a higher LBR than hormone replacement therapy (HRT) protocol accompanied by the poorest endometrial thickness [27]. Although Peigne et al. concluded similar clinical pregnancy rates $(24.4 \% \mathrm{vs}$. $20.8 \%$ ) between AC-FET and mSTC-FET groups, LBR (17.1\% vs. 9.8\%) was significantly higher with AC than with mSTC group, even after adjusting for potential bias [28]. In contrast with our findings, Azadeh et al., in a randomized clinical trial, observed no difference in pregnancy outcomes and cancellation rates between the letrozole plus HMG method and the artificial FET protocol [29]. In Yu et al.'s retrospective study, endometrial thickness was similar in AC-FET and STC-FET. The two protocols resulted in nonstatistically different rates of clinical pregnancy, ongoing pregnancy, live birth. Nevertheless, the canceled cycle rate made a significant difference [30]. Besides, two recent meta-analyses comparing L-FET with AC-FET group in PCOS patients found no difference between two groups for LBR, whereas a lower miscarriage rate was found for the L-FET than AC-FET [31, 32].

The ovarian stimulation protocol imitates the natural process of follicular development using ovulation stimulants to assist endogenous estradiol synthesis, thereby promoting endometrial growth. In this study, ovulation stimulants were letrozole combined with low doses of HMG. Letrozole is a third-generation aromatase inhibitor drug that, when used in the early stage of promoting ovulation, can decrease intraovarian and serum estrogen levels by blocking the conversion of androgens to estrogens in the ovarian granulosa cells without antagonistic effects on estrogen receptors. However, its estrogenlowering effect is significantly reduced in the late follicular stage [33]. This process led to a rapid thickening of endometrium and increased blood level in the uterus and endometrium, positively impacting pregnancy outcomes [34]. Our study revealed that the letrozole group demonstrated significantly greater endometrial thickness than the artificial cycle group prior to FET. A previous study has indicated that using letrozole for ovulation induction focused on PCOS patients improved endometrial receptivity, which might positively affect embryo implantation [35]. Likewise, another preliminary study observed that ovarian stimulation using letrozole resulted in a sevenfold increase in the expression of uterine receptivity 
markers, including integrin, leukemia inhibitory factor, and L-selectin, in women with unexplained infertility compared with spontaneous cycles [36], all of which may correlate with a higher success rate of embryo transfer. Moreover, a recent population-based study conducted in Japan indicated that letrozole administration during IVF cycles neither increased the risk of major congenital anomalies nor compromised neonatal outcomes of IVF newborns compared with natural cycles, indicating that letrozole is relatively safe [37]. Previously, studies demonstrated that the lack of a corpus luteum would perturb maternal circulation, increasing preeclampsia incidence. Mild ovarian stimulation with low doses of gonadotropins could induce the development of a proper number of follicles and ovulation, thereby ensuring better function of corpus luteum [38, 39]. However, because women with PCOS are more likely to develop OHSS than those without PCOS, women undergoing ovarian stimulation should receive a low initial dose of gonadotropins and be monitored frequently to ensure that only one or two dominant follicles are allowed to grow during endometrial preparation. Once exposed to OHSS risk or no dominant follicle, the transplant cycle would be canceled. Financial affordability is a major issue for patients seeking infertility treatment. In China, letrozole plus HMG is relatively inexpensive. Nonetheless, the ovarian stimulation protocol requires more monitoring via ultrasound examinations and endocrine, resulting in increased expenses. However, this protocol is relatively safe and is associated with increased pregnancy rates. Additional research examining the cost-effectiveness of these two regimens is required.

The artificial hormone replacement endometrial preparation protocol utilizes exogenous estradiol to simulate endocrine environment and promote endometrial development consistent with that observed in a normal menstrual cycle [40]. Although few studies demonstrate that hormone replacement therapy results in relatively unsatisfactory pregnancy outcomes, those who accept the subject point out that adverse outcomes of hormone replacement therapy cycle could be due to excessive intake of estradiol. $\mathrm{Ma}$ et al. found that uterine receptivity window remains open for a long time at low estrogen level but closes rapidly at high estrogen level [41]. Moreover, while estrogen is applied the early stage of follicles in the hormone replacement cycle, sometimes it cannot completely inhibit their development, and developing follicles can still secrete steroid hormones. The combined action of endogenous and exogenous steroid hormones may lead to imbalanced estrogen-progesterone proportion in the implant window, hence decreasing receptivity [42]. Yu et al. suggest that PCOS women and chronic anovulation may be less responsive to exogenous estradiol [30], consistent with our study that artificial hormone replacement group had a lower LBR and poorer endometrial thickness before embryo transfer. These studies may provide evidence for low LBR and high miscarriage rate in HRT group. However, the main disadvantage of this method is the adverse effects of employed hormones, such as the risk of maternal thromboembolic events and genital malformations in male fetuses [29]. Moreover, once pregnancy is confirmed, exogenous estrogen and progesterone should be used until the placenta is formed to replace the absent corpus luteum. Most importantly, exogenous hormones may be inadequate in some patients for proper endometrial development.

\section{Conclusion}


In conclusion, the mSTC-FET demonstrated a higher LBR and lowered pregnancy loss rates than the ACFET in overweight/obese women with PCOS. We acknowledge that this study has limitations. Some physicians prefer the artificial hormone replacement protocol over the ovarian stimulation protocol because it avoids inducing OHSS using gonadotropins. Although the dosage of ovulation induction drugs used in the stimulated protocol is safe, a large sample size difference was observed between the two groups. Due to the retrospective nature of this analysis, selection bias regarding the type of FET protocol may exist. However, this study also has some strengths, including a larger cohort size with a recent $100 \%$ follow-up. In addition, this study, coupled with existing evidence, confirmed that mildly stimulated cycles result in superior pregnancy outcomes than artificial cycles. These findings imply that using letrozole plus HMG for endometrial preparation might be a potentially better alternative for overweight/obese women with PCOS when considering the most cost-effective treatment with the least adverse effects on patients. To corroborate our findings, prospective randomized trials are necessary to determine the efficacy of mildly stimulated cycles as a method of endometrial preparation for FET.

\section{Abbreviations}

AC: Artificial cycles; ART: Assisted reproductive techniques; BMI: body mass index; $\mathrm{COH}$ : Controlled ovarian hyperstimulation; FET: Frozen embryo transfer; HCG: Human chorionic gonadotropin; HMG: human menopausal gonadotropin; HRT: Hormone replacement therapy; ICSI: intracytoplasmic sperm injection; IVF: in vitro fertilization; LBR: live birth rate; mSTC: mildly stimulated cycles; OHSS: ovarian hyperstimulation syndrome; OS: Ovarian stimulation; PCOS: polycystic ovary syndrome

\section{Declarations}

\section{Acknowledgements}

Not applicable

\section{Authors' contributions}

LG collected data and developed the manuscript. SX and HW guided the design and reviewed the manuscript. CP and DL contributed to data collection. CW and XY assisted in data analysis. FL guided the design and implementation of the study. All authors read and approved the final manuscript.

\section{Funding}

This study was funded by the National Natural Science Foundation of China (81974577) and the Natural Science Foundation of Shandong Province (ZR202102280473).

\section{Availability of data and materials}

The datasets used and/or analyzed in this study will be availed by the corresponding author upon a reasonable request. 


\section{Ethics approval and consent to participate}

This study was approved by the Institutional Review Board and the Institutional Ethics Committee of the Reproductive Medical Center at the affiliated Hospital of Shandong University of traditional Chinese medicine (ref approval no. SDTCM20201008). All procedures were carried out per the relevant guidelines and regulations. Furthermore, informed consent was obtained from all participants before the study.

\section{Consent for publication}

Not applicable.

\section{Competing interests}

We declare that we do not have any commercial or associative interest that represents a conflict of interest in connection with the work submitted.

\section{References}

1. Bakeer E, Radwan R, El Mandoury A, El Rahman AA, Gad M, El Maksoud SA. Anti-Müllerian Hormone as a Diagnostic Marker in Egyptian Infertile Polycystic Ovary Syndrome Females: Correlations with Vitamin D, Total Testosterone, Dyslipidemia and Anthropometric Parameters. J Med Biochem. 2018;37(4):448-455.

2. Alvarez-Blasco F, Botella-Carretero JI, San Millán JL, Escobar-Morreale HF. Prevalence and characteristics of the polycystic ovary syndrome in overweight and obese women. Arch Intern Med. 2006;166(19):2081-6.

3. Fauser BC, Tarlatzis BC, Rebar RW, Legro RS, Balen AH, Lobo R, Carmina E, Chang J, Yildiz BO, Laven JS, et al. Consensus on women's health aspects of polycystic ovary syndrome (PCOS): the Amsterdam ESHRE/ASRM-Sponsored 3rd PCOS Consensus Workshop Group. Fertil Steril. 2012;97(1):28-38.e25.

4. Li R, Zhang Q, Yang D, Li S, Lu S, Wu X, Wei Z, Song X, Wang X, Fu S, et al. Prevalence of polycystic ovary syndrome in women in China: a large community-based study. Hum Reprod. 2013;28(9):25629.

5. Lim SS, Davies MJ, Norman RJ, Moran LJ. Overweight, obesity and central obesity in women with polycystic ovary syndrome: a systematic review and meta-analysis. Hum Reprod Update. 2012;18(6):618-37.

6. Jia AH, Xu SY, Ming J, Zhou J, Guo JH, Liu C, Hao PR, Ji QH. Epidemiological characteristics of obesity under different diagnostic criteria in China. Chinese Journal of Diabetes. 2017;9(04):221225.

7. Fedorcsák P, Storeng R, Dale PO, Tanbo T, Abyholm T. Obesity is a risk factor for early pregnancy loss after IVF or ICSI. Acta Obstet Gynecol Scand. 2000;79(1):43-8. PMID: 10646815. 
8. Turhan NO, Seçkin NC, Aybar F, Inegöl I. Assessment of glucose tolerance and pregnancy outcome of polycystic ovary patients. Int J Gynaecol Obstet. 2003 May;81(2):163-8.

9. Balen AH, Morley LC, Misso M, Franks S, Legro RS, Wijeyaratne CN, Stener-Victorin E, Fauser BC, Norman RJ, Teede $\mathrm{H}$. The management of anovulatory infertility in women with polycystic ovary syndrome: an analysis of the evidence to support the development of global WHO guidance. Hum Reprod Update. 2016;22(6):687-708.

10. Wei YM, Yang HX, Zhu WW, Liu XY, Meng WY, Wang YQ, Shang LX, Cai ZY, Ji LP, Wang YF, et al. Risk of adverse pregnancy outcomes stratified for pre-pregnancy body mass index. J Matern Fetal Neonatal Med. 2016;29(13):2205-9.

11. Zech J, Brandao A, Zech M, Lugger K, Neururer S, Ulmer H, Ruttmann-Ulmer E. Elective frozen-thawed embryo transfer (FET) in women at risk for ovarian hyperstimulation syndrome. Reprod Biol. 2018;18(1):46-52.

12. Roque $M$, Valle $M$, Guimarães $F$, Sampaio $M$, Geber S. Freeze-all policy: fresh vs. frozen-thawed embryo transfer. Fertil Steril. 2015;103(5):1190-1193.

13. Barnhart KT. Introduction: are we ready to eliminate the transfer of fresh embryos in in vitro fertilization? Fertil Steril. 2014;102(1):1-2.

14. Shapiro BS, Daneshmand ST, Garner FC, Aguirre M, Hudson C, Thomas S. Evidence of impaired endometrial receptivity after ovarian stimulation for in vitro fertilization: a prospective randomized trial comparing fresh and frozen-thawed embryo transfer in normal responders. Fertil Steril. 2011;96(2):344-8.

15. Mackens S, Santos-Ribeiro S, van de Vijver A, Racca A, Van Landuyt L, Tournaye H, Blockeel C. Frozen embryo transfer: a review on the optimal endometrial preparation and timing. Hum Reprod. 2017;32(11):2234-2242.

16. Montagut M, Santos-Ribeiro S, De Vos M, Polyzos N, Drakopoulos P, Mackens S, van de Vijver A, van Landuyt L, Verheyen G, Tournaye H, et al. Frozen-thawed embryo transfers in natural cycles with spontaneous or induced ovulation: the search for the best protocol continues. Hum Reprod. 2016;31(12):2803-2810.

17. Chen R, Chen S, Liu M, He H, Xu H, Liu H, Du H, Wang W, Xia X, Liu J. Pregnancy outcomes of PCOS overweight/obese patients after controlled ovarian stimulation with the $\mathrm{GnRH}$ antagonist protocol and frozen embryo transfer. Reprod Biol Endocrinol. 2018;16(1):36.

18. Rotterdam ESHRE/ASRM-Sponsored PCOS Consensus Workshop Group. Revised 2003 consensus on diagnostic criteria and long-term health risks related to polycystic ovary syndrome. Fertil Steril. 2004;81(1):19-25.

19. Cummins JM, Breen TM, Harrison KL, Shaw JM, Wilson LM, Hennessey JF. A formula for scoring human embryo growth rates in in vitro fertilization: its value in predicting pregnancy and in comparison with visual estimates of embryo quality. J In Vitro Fert Embryo Transf. 1986;3(5):28495. 
20. Gardner D, Lane M, Stevens J, Schlenker T, Schoolcraft W. Blastocyst score affects implantation and pregnancy outcome: towards a single blastocyst transfer. Fertil Steril. 2000;73(6):1155-1158.

21. Song J, Xiang S, Sun Z. Frozen embryo transfer at the cleavage stage can be performed within the first menstrual cycle following the freeze-all strategy without adversely affecting the live birth rate: A STROBE-compliant retrospective study. Medicine. 2019;98(38):e17329.

22. Lin J, Huang J, Wang N, Kuang Y, Cai R. Effects of pre-pregnancy body mass index on pregnancy and perinatal outcomes in women with PCOS undergoing frozen embryo transfer. BMC Pregnancy Childbirth. 2019;19(1):487.

23. Mu L, Zhao Y, Qiao J. Relationship between obesity and polycystic ovary syndrome and its potential mechanisms. Chin J Reprod Contracep. 2018;38(04):343-349

24. Zhang J, Liu H, Wang Y, Mao X, Chen Q, Fan Y, Xiao Y, Kuang Y. Letrozole use during frozen embryo transfer cycles in women with polycystic ovary syndrome. Fertil Steril. 2019;112(2):371-377.

25. Tatsumi T, Jwa SC, Kuwahara A, Irahara M, Kubota T, Saito H. Pregnancy and neonatal outcomes following letrozole use in frozen-thawed single embryo transfer cycles. Hum Reprod. 2017;32(6):1244-1248.

26. Aslih N, Dorzia D, Atzmon Y, Estrada D, Ellenbogen A, Bilgory A, Shalom-Paz E. Ovulatory-Based FET Cycles May Achieve Higher Pregnancy Rates in the General Population and among Anovulatory Women. J Clin Med. 2021;10(4):703.

27. Man Y, Bian Y, Zhao S, Zhao R, Xu X, Wei D, Li L, Chen ZJ, Zhao H. The effect of different endometrial preparations on women with polycystic ovary syndrome undergoing initial frozen embryo transfer: $A$ historical cohort analysis. Acta Obstet Gynecol Scand. 2021;100(6):1116-1123.

28. Peigné M, Devouche E, Ferraretto X, Gricourt S, Luton D, Patrat C, Epelboin S. Higher live birth rate with stimulated rather than artificial cycle for frozen-thawed embryo transfer. Eur J Obstet Gynecol Reprod Biol. 2019;243:144-149.

29. Hosseini-Najarkolaei A, Moini A, Kashani L, Farid Mojtahedi M, Hosseini-Najarkolaee E, Salehi E. The effect of letrozole versus artificial hormonal endometrial preparation on pregnancy outcome after frozen-thawed embryos transfer cycles: a randomized clinical trial. Reprod Biol Endocrinol. 2020;18(1):115.

30. Yu J, Ma Y, Wu Z, Li Y, Tang L, Li Y, Deng B. Endometrial preparation protocol of the frozen-thawed embryo transfer in patients with polycystic ovary syndrome. Arch Gynecol Obstet. 2015;291(1):201211.

31. Zeng MF, Zhou X, Duan JL. Stimulated cycle versus artificial cycle for frozen embryo transfer in patients with polycystic ovary syndrome: a Meta-analysis. Gynecol Endocrinol. 2021;37(4):294-299.

32. Chen D, Shen X, Fu Y, Ding C, Zhong Y, Zhou C. Pregnancy Outcomes Following Letrozole Use in Frozen-thawed Embryo Transfer Cycles: A Systematic Review and Meta-analysis. Geburtshilfe Frauenheilkd. 2020;80(8):820-833.

33. Kar S. Clomiphene citrate or letrozole as first-line ovulation induction drug in infertile PCOS women: A prospective randomized trial. J Hum Reprod Sci. 2012;5(3):262-5. 
34. Hu YJ, Chen YZ, Zhu YM, Huang HF. Letrozole stimulation in endometrial preparation for cryopreserved-thawed embryo transfer in women with polycystic ovarian syndrome: a pilot study. Clin Endocrinol (Oxf). 2014;80(2):283-9.

35. Wallace K, Johnson V, Sopelak V, Hines R. Clomiphene citrate versus letrozole: molecular analysis of the endometrium in women with polycystic ovary syndrome. Fertil Steril. 2011;96(4):1051-1056.

36. Ganesh A, Chauhan N, Das S, Chakravarty B, Chaudhury K. Endometrial receptivity markers in infertile women stimulated with letrozole compared with clomiphene citrate and natural cycles. Syst Biol Reprod Med. 2014;60(2):105-111.

37. Tatsumi T, Jwa SC, Kuwahara A, Irahara M, Kubota T, Saito H. No increased risk of major congenital anomalies or adverse pregnancy or neonatal outcomes following letrozole use in assisted reproductive technology. Hum Reprod. 2017;32(1):125-132.

38. von Versen-Höynck F, Schaub AM, Chi YY, Chiu KH, Liu J, Lingis M, Stan Williams R, Rhoton-Vlasak A, Nichols WW, Fleischmann RR, et al. Increased Preeclampsia Risk and Reduced Aortic Compliance With In Vitro Fertilization Cycles in the Absence of a Corpus Luteum. Hypertension. 2019;73(3):640649.

39. Conrad KP, Petersen JW, Chi YY, Zhai X, Li M, Chiu KH, Liu J, Lingis MD, Williams RS, Rhoton-Vlasak A, et al. Maternal Cardiovascular Dysregulation During Early Pregnancy After In Vitro Fertilization Cycles in the Absence of a Corpus Luteum. Hypertension. 2019;74(3):705-715.

40. Groenewoud ER, Cohlen BJ, Macklon NS. Programming the endometrium for deferred transfer of cryopreserved embryos: hormone replacement versus modified natural cycles. Fertil Steril. 2018;109(5):768-774.

41. Ma WG, Song H, Das SK, Paria BC, Dey SK. Estrogen is a critical determinant that specifies the duration of the window of uterine receptivity for implantation. Proc Natl Acad Sci U S A. 2003;100(5):2963-8.

42. Jiang $C$, Zhang $C$. Comparing the effection of endometrial preparation protocols of hormone replacement treatment with or without pituitary suppression on the outcomes of frozen thawed embryo transfer cycles. Prog Obstet Gynecol. 2015;24(06):441-443.

43. Groenewoud ER, Cohlen BJ, Al-Oraiby A, Brinkhuis EA, Broekmans FJ, de Bruin JP, van den Dool G, Fleisher K, Friederich J, Goddijn M, et al. A randomized controlled, non-inferiority trial of modified natural versus artificial cycle for cryo-thawed embryo transfer. Hum Reprod. 2016;31(7):1483-92.

\section{Tables}

Table 1. Baseline characteristics of ovarian stimulation and IVF cycles. 


\begin{tabular}{|c|c|c|c|c|}
\hline & Total & mSTC & $\mathrm{AC}$ & $\begin{array}{l}\mathrm{P} \\
\text { value }\end{array}$ \\
\hline Number of patients & 680 & 173 & 507 & \\
\hline Age of woman (years) & $30.10 \pm 3.22$ & $30.31 \pm 3.49$ & $30.20 \pm 3.12$ & 0.346 \\
\hline Infertility duration(years) & $3.25 \pm 2.03$ & $3.05 \pm 1.97$ & $3.32 \pm 2.05$ & 0.122 \\
\hline BMI $\left(\mathrm{kg} / \mathrm{m}^{2}\right)$ & $27.77 \pm 4.33$ & $27.40 \pm 4.19$ & $27.90 \pm 4.37$ & 0.122 \\
\hline \multicolumn{5}{|l|}{ Baseline hormonal profile } \\
\hline $\mathrm{FSH}(\mathrm{mlU} / \mathrm{mL})$ & $6.40 \pm 1.56$ & $6.39 \pm 1.67$ & $6.40 \pm 1.52$ & 0.982 \\
\hline $\mathrm{LH}(\mathrm{mlU} / \mathrm{mL})$ & $8.32 \pm 4.60$ & $8.58 \pm 5.01$ & $8.22 \pm 4.45$ & 0.374 \\
\hline Type of infertility & & & & 0.514 \\
\hline Primary infertility & $380(55.9 \%)$ & $93(53.8 \%)$ & $287(56.6 \%)$ & \\
\hline Secondary infertility & $300(54.1 \%)$ & $80(46.2 \%)$ & $220(43.4 \%)$ & \\
\hline Laboratory insemination & & & & 0.162 \\
\hline IVF & $573(85.7 \%)$ & $140(80.9 \%)$ & $433(87.4 \%)$ & \\
\hline ICSI & 107(14.3\%) & $33(19.1 \%)$ & $74(12.6 \%)$ & \\
\hline Ovulation induction regimen & & & & 0.406 \\
\hline Agonist regimen & $496(72.9 \%)$ & $122(70.5 \%)$ & $374(73.8 \%)$ & \\
\hline Antagonist regimen & 184(27.1\%) & $51(29.5 \%)$ & $133(26.2 \%)$ & \\
\hline Gn days (days) & $11.36 \pm 2.96$ & $11.58 \pm 3.06$ & $11.29 \pm 2.92$ & 0.262 \\
\hline Total Gn (mlU/mL) & $2287.28 \pm 1001.74$ & $2388.54 \pm 1031.18$ & $2252.73 \pm 990.16$ & 0.124 \\
\hline Number of eggs obtained & $22.26 \pm 9.48$ & $24.40 \pm 4.19$ & $22.13 \pm 9.614$ & 0.189 \\
\hline $\begin{array}{l}\text { Total number of frozen } \\
\text { embryos }\end{array}$ & $6.94 \pm 2.36$ & $7.18 \pm 2.43$ & $6.85 \pm 2.33$ & 0.107 \\
\hline
\end{tabular}

Note: Data are presented as mean \pm SD for continuous variables and $\mathrm{n}(\%)$ for dichotomous variables. All $P$ values were assessed with the use of student's t-test or $\chi^{2}$.

$\mathrm{BMI}=$ body mass index; $\mathrm{FSH}=$ follicle stimulating hormone; $\mathrm{Gn}=$ gonadotropin; ICSI=intracytoplasmic sperm injection; IVF=in vitro fertilization; LH=luteinizing hormone.

\section{Table 2. FET cycle characteristics between groups.}

Note: Data are presented as mean \pm SD for continuous variables and $\mathrm{n}(\%)$ for dichotomous variables. All $P$ values were assessed with the use of student's t-test or $\chi^{2}$. 


\begin{tabular}{|lllll|}
\hline & Total & mSTC & AC & P value \\
\hline Number of patients & 680 & 173 & 507 & \\
\hline Number of embryos transferred & & & & 0.457 \\
\hline Single & $94(13.8 \%)$ & $21(12.1 \%)$ & $73(14.4 \%)$ & \\
\hline Double & $586(86.2 \%)$ & $152(87.9 \%)$ & $434(85.6 \%)$ & \\
\hline Embryo grades & & & & 0.382 \\
\hline Level 1 & $446(35.2 \%)$ & $108(33.2 \%)$ & $338(36.0 \%)$ & \\
\hline Level 2 & $820(64.8 \%)$ & $217(66.8 \%)$ & $603(64.0 \%)$ & \\
\hline Embryos stages & & & & 0.402 \\
\hline Day 3 embryos & $597(87.8 \%)$ & $155(89.6 \%)$ & $442(87.2 \%)$ & \\
\hline Day 5 embryos & $83(12.2 \%)$ & $18(10.4 \%)$ & $65(12.8 \%)$ & \\
\hline Endometrium thickness prior to FET (mm) & $9.84 \pm 1.71$ & $10.10 \pm 1.94$ & $9.75 \pm 1.61$ & 0.039 \\
\hline
\end{tabular}

Table 3. Pregnancy outcomes between groups.

\begin{tabular}{|lllll|}
\hline & Total & mSTC & AC & P value \\
\hline Number of patients & 680 & 173 & 507 & $\begin{array}{l}\text { Note: } \\
\text { Data } \\
\text { are }\end{array}$ \\
\hline Implantation rate & $458 / 1266(36.2 \%)$ & $111 / 325(34.2 \%)$ & $347 / 941(36.9 \%)$ & 0.379 \\
\hline Positive pregnancy rate & $404 / 680(59.4 \%)$ & $100 / 173(57.8 \%)$ & $304 / 507(60.0 \%)$ & 0.618 \\
\hline Clinical pregnancy rate & $376 / 680(55.3 \%)$ & $94 / 173(54.3 \%)$ & $282 / 507(55.6 \%)$ & 0.769 \\
\hline Twin pregnancies & $69 / 376(18.4 \%)$ & $19 / 94(20.2 \%)$ & $70 / 282(24.8 \%)$ & 0.362 \\
\hline Ectopic pregnancy & $11 / 376(3.0 \%)$ & $2 / 94(2.1 \%)$ & $9 / 282(3.2 \%)$ & 0.860 \\
\hline Miscarriage rate & $68 / 376(18.1 \%)$ & $6 / 94(6.4 \%)$ & $65 / 282(23.0 \%)$ & $<0.001$ \\
\hline Live birth rate & $294 / 680(43.2 \%)$ & $86 / 173(49.7 \%)$ & $208 / 507(41.0 \%)$ & 0.046 \\
\hline Singletons & $235 / 680(34.6 \%)$ & $70 / 173(40.5 \%)$ & $165 / 507(32.5 \%)$ & 0.059 \\
\hline Twins & $59 / 680(8.7 \%)$ & $16 / 173(9.2 \%)$ & $43 / 507(8.5 \%)$ & 0.757 \\
\hline
\end{tabular}

presented as mean \pm SD for continuous variables and $\mathrm{n}(\%)$ for dichotomous variables. All $P$ values were assessed with the use of student's t-test or $\chi^{2}$.

Table 4. Binary logistics regression analysis with live birth rate as the influencing factor.

Note: Analyses were adjusted for age at FET, body mass index (BMI), infertility duration, infertility type (primary, secondary), ovulation induction regimen (Agonist regimen, Antagonist regimen), Gn usage time, 


\begin{tabular}{|c|c|c|c|c|c|}
\hline \multirow{2}{*}{$\begin{array}{l}\text { Pregnancy } \\
\text { Outcomes }\end{array}$} & \multirow{2}{*}{$\begin{array}{l}\text { Endometrial } \\
\text { Preparation }\end{array}$} & \multicolumn{2}{|l|}{ Crude Model } & \multicolumn{2}{|c|}{ Adjusted Model } \\
\hline & & OR(95\% Cl) & $\begin{array}{l}\mathrm{P} \\
\text { value }\end{array}$ & $\mathrm{OR}(95 \% \mathrm{Cl})$ & $\begin{array}{l}P \\
\text { value }\end{array}$ \\
\hline \multirow{2}{*}{$\begin{array}{l}\text { Positive pregnancy } \\
\text { rate }\end{array}$} & mSTC & \multirow{2}{*}{$\begin{array}{l}0.915(0.645- \\
1.298)\end{array}$} & \multirow[t]{2}{*}{0.618} & \multirow{2}{*}{$\begin{array}{l}0.920(0.646- \\
1.312)\end{array}$} & \multirow[t]{2}{*}{0.646} \\
\hline & $A C$ & & & & \\
\hline \multirow{2}{*}{$\begin{array}{l}\text { Clinical pregnancy } \\
\text { rate }\end{array}$} & mSTC & \multirow{2}{*}{$\begin{array}{l}0.949(0.671- \\
1.343)\end{array}$} & \multirow[t]{2}{*}{0.769} & \multirow{2}{*}{$\begin{array}{l}0.950(0.669- \\
1.351)\end{array}$} & \multirow[t]{2}{*}{0.777} \\
\hline & $A C$ & & & & \\
\hline \multirow[t]{2}{*}{ Miscarriage rate } & mSTC & \multirow{2}{*}{$\begin{array}{l}0.244(0.104- \\
0.574)\end{array}$} & \multirow[t]{2}{*}{$<0.001$} & \multirow{2}{*}{$\begin{array}{l}0.237(0.100- \\
0.562)\end{array}$} & \multirow[t]{2}{*}{$<0.001$} \\
\hline & $A C$ & & & & \\
\hline \multirow[t]{2}{*}{ Live birth rate } & mSTC & \multirow{2}{*}{$\begin{array}{l}1.393(0.986- \\
1.970)\end{array}$} & \multirow[t]{2}{*}{0.046} & \multirow{2}{*}{$\begin{array}{l}1.462(1.028- \\
2.079)\end{array}$} & \multirow[t]{2}{*}{0.035} \\
\hline & $A C$ & & & & \\
\hline
\end{tabular}

Gn dosage, oocytes retrieved, laboratory fertilization mode (IVF, ICSI), number of embryos transferred, embryo stage and quality at transfer, endometrium thickness prior to FET,

\section{Figures}

\section{Figure 1}

Workflow of study enrollment. 